\title{
WG1: Structure Functions and Parton Densities
}

\author{
Lucian Harland-Lang* \\ Rudolf Peierls Centre for Theoretical Physics, Oxford, UK \\ E-mail: lucian.harland-lang@physics.ox.ac.uk
}

\section{Philip Ilten}

School of Physics and Astronomy, University of Birmingham, Birmingham, UK

E-mail: philten@cern.ch

\section{Jan Kretzschmar}

Oliver Lodge Laboratory, University of Liverpool, Liverpool, UK

E-mail: jan.kretzschmarecern.ch

\begin{abstract}
This paper gives a summary of selected highlights discussed in the working group on "Structure Functions and Parton Densities" (WG1) at the DIS 2017 conference. From the many talks presented we extract some general themes discussed with respect to global PDF fits, new PDFsensitive measurements from the LHC experiments and elsewhere, exploitation of new ideas, tools to perform PDF fits, PDFs of heavy nuclei, and finally progress in basic theory calculations.
\end{abstract}

XXV International Workshop on Deep-Inelastic Scattering and Related Subjects 3-7 April 2017

University of Birmingham, UK

\footnotetext{
* Speaker.
} 


\section{Introduction}

The "Structure Functions and Parton Densities" working group (WG1) of the DIS 2017 conference featured 45 talks spanning a wide range of interesting topics. The talks were organised in 10 sessions, including one joint session with "Physics with Heavy Flavours" (WG5) [1] and two joint sessions with "Hadronic and Electroweak Observables" (WG4) [2]. In the following we summarise some selected highlights of these sessions.

\section{Global PDF Fits}

Updates from three major global fitting collaborations CT [3], MMHT [4], and NNPDF [5] were presented. In all cases, the results shown made use of an increasingly wide range of data from the LHC experiments and the LHC data are playing an increasingly important role in global PDF fits. Studies beyond the new LHC data were also presented, for example regarding updates on the photon PDF from the CT and MMHT groups and intrinsic charm by the CT and NNPDF groups, as discussed further below. Work towards new public releases of PDF sets is ongoing for the CT and MMHT groups, while the updated NNPDF3.1 set based on the work presented here is now available [6].

In Fig. 1 (left) the impact of new data on the gluon, predominately from the LHC, is shown by comparing the new determination to the previous NNPDF3.0 determination; the changes are significant, especially at larger $x$. A similar effect is observed by CT and MMHT. In the latter case, for example, LHC data is found to provide the most important constraints in the latest fit for 21 out of the 50 eigenvector directions. In Fig. 1 (right) the constraint that the new high precision ATLAS $W$ and $Z$ production data [7, 8] places on the strange-quark distribution in the proton (the ratio of the strange to the light-quark sea is shown here) is demonstrated, within the MMHT fit. The impact is large, with the strange-quark fraction found to be somewhat higher than the previous determination, although consistent, and the uncertainties are reduced. A similar trend is seen in the NNPDF analysis.

LHC data certainly represent important opportunities to improve the knowledge on PDFs. However, there are many challenges for the PDF fitters to include such increasingly precise data effectively within a PDF fit. Some of these challenges were also highlighted in this conference, and will be discussed below.

\section{New Measurements sensitive to PDFs and $\alpha_{S}$}

There has been significant progress in the past year regarding new experimental measurements. The majority of new results were presented by the ATLAS, CMS, and LHCb collaborations at the LHC, but new results are still being made public from the HERA experiments like H1. These data can be used to further constrain PDFs and measure the strong coupling $\alpha_{S}$. The results span a wide range of physical observables including jets, vector bosons in association with heavy flavour jets, and high-precision inclusive vector-boson measurements.

Double-differential jet cross sections in DIS have been measured as a function of virtuality $Q^{2}$ and jet $p_{\mathrm{T}}$ by the H1 collaboration $[9,10]$. These data have been compared to the latest nextto-next-to-leading order (NNLO) theory calculations for the first time for a $Q^{2}$ range of 5.5 to 

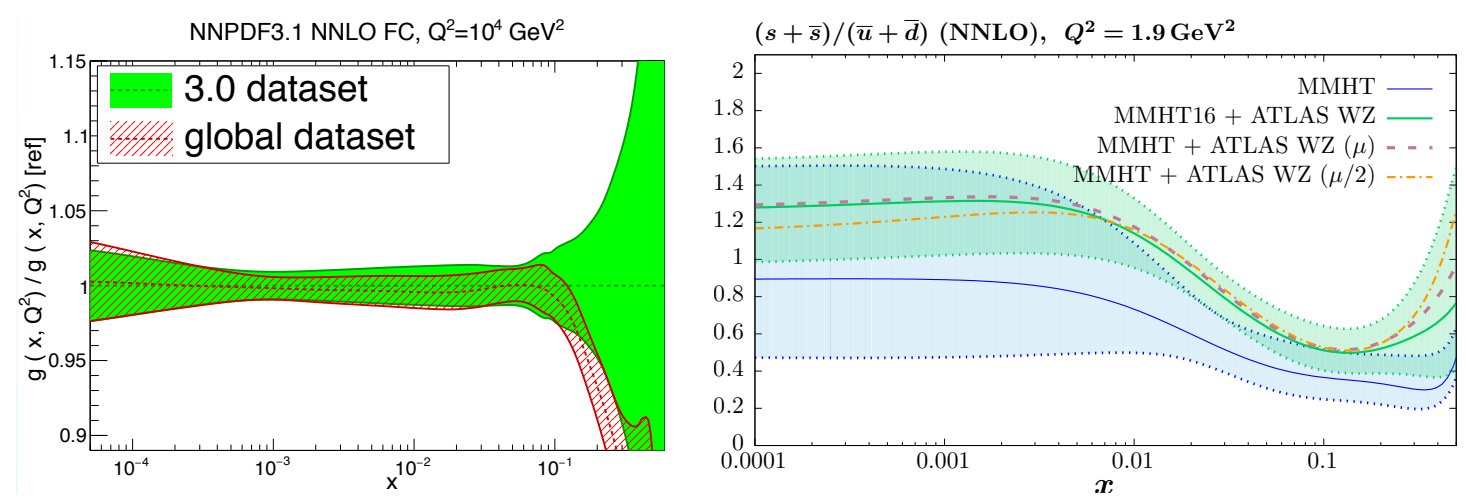

Figure 1: (Left) Impact of new data on the gluon PDF, predominately from the LHC, in the new NNPDF3.1 fit as compared to the previous NNPDF3.0 analysis [5]. (Right) Impact of the high precision 7 TeV ATLAS $W$ and $Z$ production data on the strange-quark contribution to the proton in the MMHT fit [4].

$15000 \mathrm{GeV}^{2}$ and a jet $p_{\mathrm{T}}$ range of 5 to $40 \mathrm{GeV}$. As seen in Fig. 2 (left), the NNLO predictions provide a better description of the cross section shape as compared to NLO predictions, as well as a reduction in the theoretical uncertainties as estimated by scale variations. Double-differential inclusive jet cross sections in $p p$ collisions at $8 \mathrm{TeV}$ in jet $y$ and $p_{\mathrm{T}}$ have been performed by the ATLAS and CMS collaborations [11, 12, 13, 14, 15]. The CMS result also provides ratios between the cross sections measured at $\sqrt{s}=8$ and $2.76 \mathrm{TeV}$ [15], resulting in a partial cancellation of systematic uncertainties and greater sensitivity to PDFs. As shown in Fig. 2 (right), the HERAPDF1.5 and MMHT14 PDF sets describe this ratio well, while the ABM11, CT10, and NNPDF3.0 PDF sets systematically under-estimate the cross section ratio at lower jet $p_{\mathrm{T}}$. Analyses of the ATLAS measurement at $\sqrt{s}=8 \mathrm{TeV}$ [14] within the framework of global PDF fits have reported difficulties in describing the measurement across all jet $y$ bins, similar to the previous $\sqrt{s}=7 \mathrm{TeV}$ ATLAS measurement [16]. However, decorrelating two of the jet energy scale systematics has been found to significantly improve the description of the $7 \mathrm{TeV}$ data $[4,17]$. Further studies of the $8 \mathrm{TeV}$ data are therefore required to fully assess its impact on PDFs.

Differential cross section measurements for dijet and trijet events have also been made by the $\mathrm{H} 1$ and CMS collaborations $[9,10,12,13,18,15]$. The H1 dijet results are again compared to NNLO theory predictions. While the NNLO predictions describe the shape well, they consistently overestimate the cross section. The $\mathrm{H} 1$ trijet measurement is more limited by statistical uncertainties and found to be in good agreement with the NLO predictions. A ratio between the CMS inclusive trijet and dijet cross sections at $\sqrt{s}=8 \mathrm{TeV}$ as a function of the average $p_{\mathrm{T}}$ of the two highest $p_{\mathrm{T}}$ jets in the event is particularly sensitive to $\alpha_{s}$ [12,19]. Fits of this ratio, as well as the dijet and trijet cross sections, were performed using a variety of PDF sets to determine $\alpha_{S}\left(M_{z}\right)$. The extraction of $\alpha_{S}\left(M_{Z}\right)$ from the ratio fit typically resulted in lower values of $\alpha_{S}\left(M_{z}\right)$, giving $0.1139 \pm 0.0032$ with $\mathrm{CT} 14$, than the simultaneous dijet and trijet cross section fits, which gives $0.1161 \pm 0.0029$ with CT14, where the quoted uncertainties exclude QCD scale variations. Electroweak corrections were calculated for the dijet fits but not the trijet fits. However, these corrections are assumed to have a minor effect in the cross section ratio, and so the $\alpha_{S}\left(M_{Z}\right)$ value extracted from the ratio is considered as the nominal result in this analysis, leading to the central 

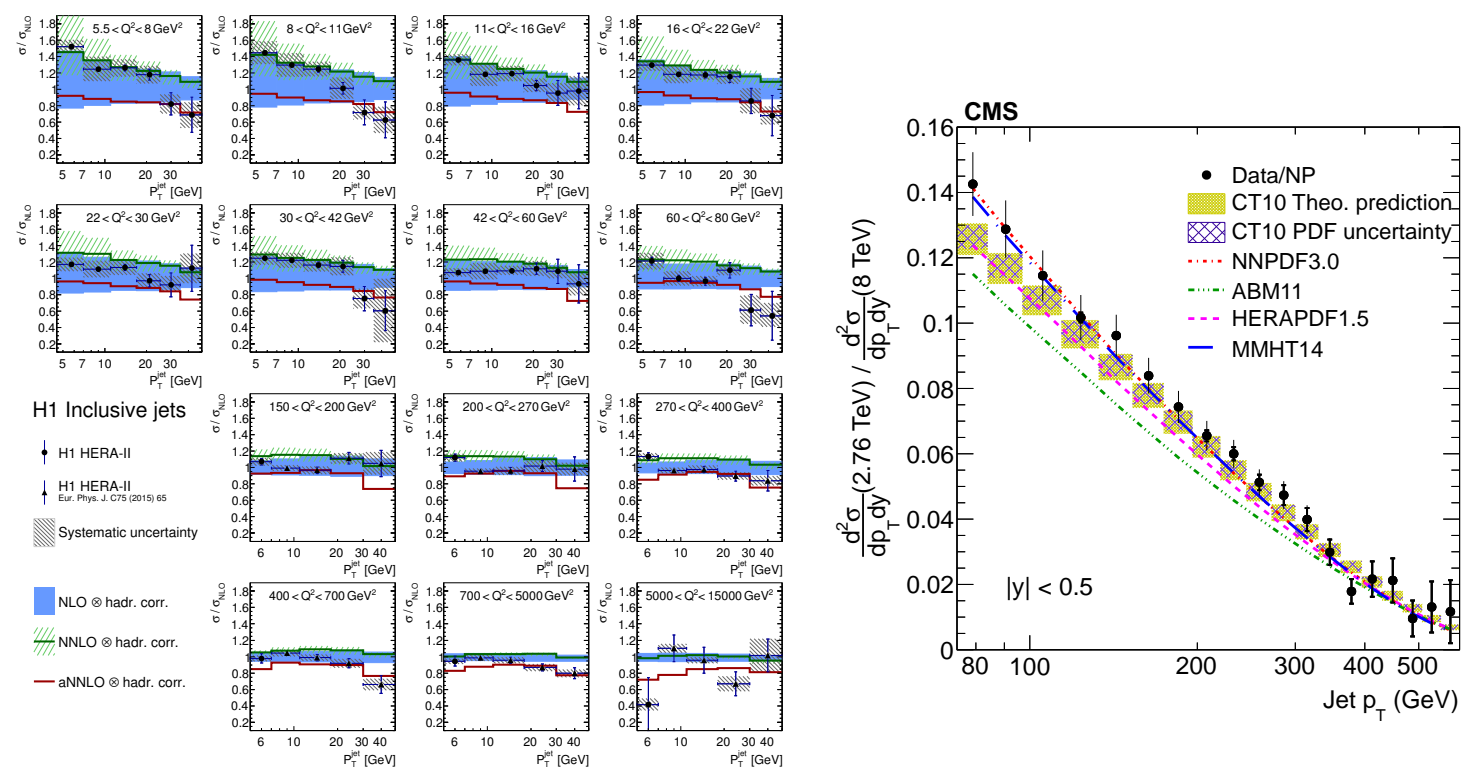

Figure 2: (Left) Inclusive differential jet cross sections from H1 [9]. (Right) Ratio between the inclusive jet cross sections at $\sqrt{s}=2.76$ and $8 \mathrm{TeV}$ from CMS [15].

result of $\alpha_{S}\left(M_{z}\right)=0.1150 \pm 0.0023_{-0.0000}^{+0.0050}$ using the MSTW2008 PDF set without LHC jet data. The second uncertainty is from QCD scale variations while the the first includes all other remaining uncertainties.

Larger datasets and progress in tagging of heavy-flavour jets has allowed new measurements of vector bosons in association with heavy flavour jets from both CMS and LHCb to be made. The CMS collaboration presented differential $Z+c$ cross sections measured at $\sqrt{s}=8 \mathrm{TeV}$ as a function of the $p_{\mathrm{T}}$ for both the $Z$-boson and $c$-jet [20,21]. This is in principle sensitive to intrinsic charm contributions to the PDFs, but so far large statistical and $c$-tagging efficiency uncertainties do not allow for strong constraints. The differential $Z+c$ and ratio of $Z+c$ to $Z+b$ cross sections are compared to theory predictions from MADGRAPH, aMC@NLO, and MCFM in Fig. 3. While the MADGRAPH and aMC@NLO predictions are found to be in good agreement with data, the MCFM predictions are consistently lower than the measured result, independent of the PDF set used, and further work is required to fully understand the implications of this measurement. A combined measurement of the $W+c \bar{c}, W+b \bar{b}$, and $t \bar{t}$ cross sections was performed by LHCb [22] using $\sqrt{s}=8 \mathrm{TeV}$ data with an electron or muon final state in association with two jets tagged to contain heavy-flavour hadrons. While this measurement is statistically limited, future updates will provide valuable information on the strange-quark PDF. Further measurements of the $W+c$ final state by the ATLAS, CMS, and LHCb collaborations will help constrain the size and possible asymmetry of the strange-quark PDF, and resolve the current mild tensions between different datasets.

New precise differential measurements of inclusive vector-boson production have been made by the ATLAS, LHCb, and STAR collaborations. Integrated and differential $W$ and $\gamma^{*} / Z$ cross sections at $\sqrt{s}=7 \mathrm{TeV}$ were measured by the ATLAS collaboration $[7,8]$ using both electron and muon final states with systematic uncertainties well below the percent level and negligible statistical uncertainty, testing lepton universality at the percent level. A PDF analysis was performed using 

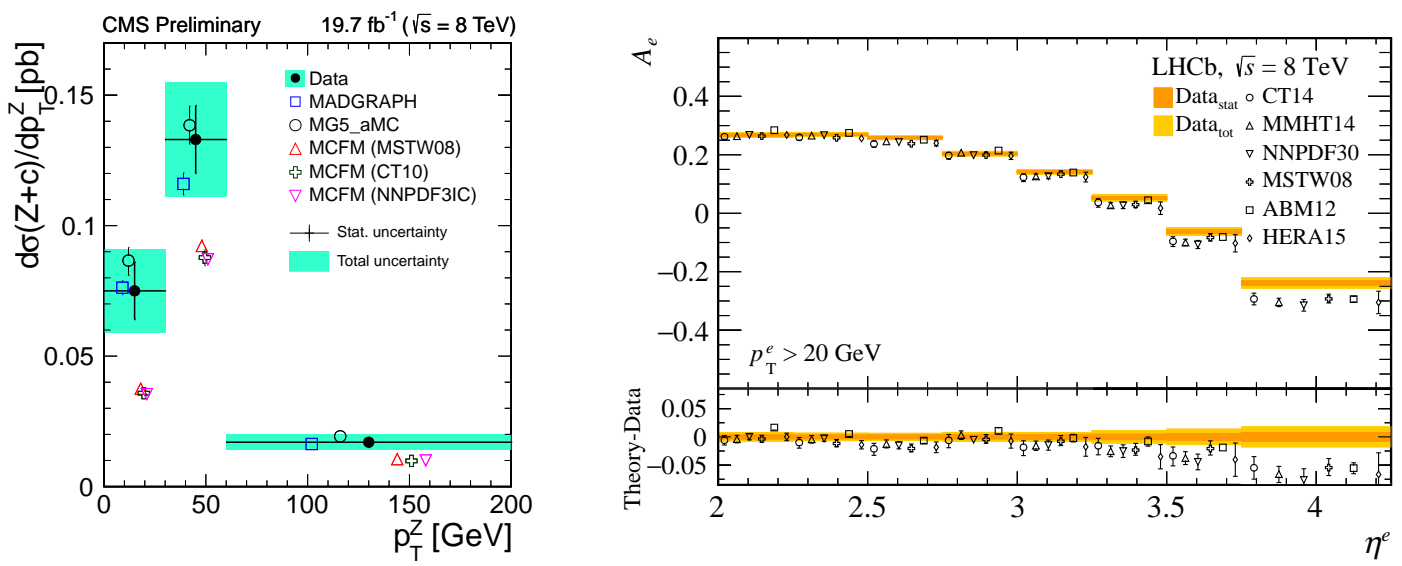

Figure 3: (Left) Differential $Z+c$ cross section measured by CMS [21]. (Right) Charge asymmetry for $W \rightarrow e$ measured as a function of electron pseudorapidity from $\mathrm{LHCb}$ [23].
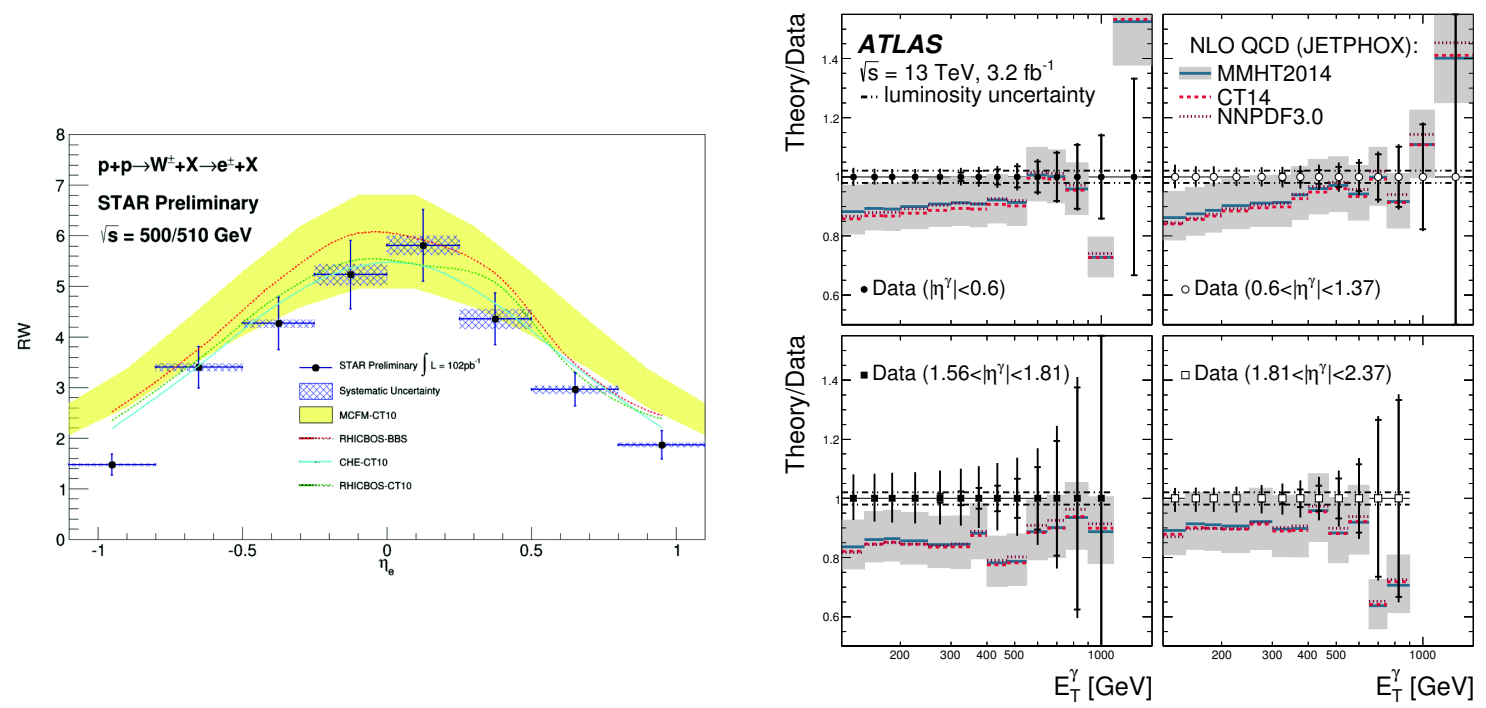

Figure 4: (Left) Differential $W^{+} / W^{-}$cross section ratio from STAR as a function of electron pseudorapidity. (Right) Isolated photon cross section from ATLAS compared to NLO theory predictions.

these data combined with HERA data, and the strange-to-light sea-quark ratio was found to be near unity, a result confirmed in global PDF analyses performed by MMHT [4] and NNPDF [5], albeit with a reduced fit quality. The MMHT study and an ATLAS study demonstrated that halving the nominal factorisation and renormalisation scale, the di-lepton mass, improves the fit quality but has only a small impact on the strange-quark PDF [4, 8]. New ATLAS measurements of highmass Drell-Yan cross sections were presented in [24, 25], which are generally well described by different PDF sets, but were found to reduce significantly the variations of the photon PDF in the NNPDF2.3QED set.

Similar precision measurements of $\gamma^{*} / Z$ at $\sqrt{s}=13 \mathrm{TeV}$ and $W$ at $\sqrt{s}=8 \mathrm{TeV}$ were performed by LHCb [26, 23, 27], which is sensitive to PDFs at both lower and higher $x$ due to the 
forward rapidity coverage of the detector. These results were found to be in good agreement with NNLO predictions using a variety of PDF sets. However, the charge asymmetry at forward lepton pseudorapidity was found to be systematically underestimated by all PDF sets tested, see Fig. 3 (right). The $W^{+} / W^{-}$ratio using $W$-boson decays into an electron final state was measured differentially in pseudorapidity by the STAR collaboration using RHIC $p p$ data at $\sqrt{s}$ between 500 and $510 \mathrm{GeV}$ [28]. The results are shown in Fig. 4 (left), where a slight tension between theory and data at large and small electron pseudorapidity is visible.

Finally, measurements of isolated photon production differential in photon $E_{\mathrm{T}}$ and pseudorapidity, were performed by ATLAS at $\sqrt{s}=13 \mathrm{TeV}[29,30]$. The NLO predictions were found to systematically underestimate the photon cross section, particularly at lower $E_{\mathrm{T}}$ as shown in Fig. 4 (right), demonstrating the need for NNLO predictions that have recently become available [31].

\section{New Ideas to constrain PDFs}

While many of the discussions in WG1 were centred around existing fit frameworks and the inclusion of data traditionally used to constrain PDFs, many new ideas and approaches were also discussed. Novel possibilities to constrain the gluon PDF at low $x$ were introduced in [32] and [33]. In $[32,34]$, data of open-charm hadron production from $p p$ collisions in the forward region taken by the LHCb experiment was demonstrated to have significant constraining potential. These constraints have implications for the production of neutrinos though heavy-flavour production in ultra high-energy cosmic ray collisions and for physics at a possible $\sqrt{s}=100 \mathrm{TeV}$ FCC. In $[33,35]$ the exclusive photoproduction of heavy quarkonia $(J / \psi, \Upsilon)$, where both protons remain intact after the collision, was also shown to have a significant impact on the gluon PDF in the very low $x$ region. However, both processes are affected by large theoretical uncertainties estimated from varying the factorisation and renormalisation scales. In [32] the impact was minimised by choosing suitably normalised observables and assuming that these variations are largely correlated, while in [33] a physically motivated scale choice was taken.

The high $x$ gluon was considered in two separate contributions, [36] and [37]. In [36] a PDF fit was described that utilises the new NNLO QCD theory calculation combined with the recent ATLAS and CMS data for differential top-quark production; these measurements are differential in $m_{t \bar{t}}, y_{t}, y_{t \bar{t}}$ and $p_{\mathrm{T}}^{t}$, see [38] for details. Although there are tensions in some distributions between the ATLAS and CMS data, the impact on the gluon distribution of the individual distribution choices was mostly consistent. A recommendation was made to use a combination of the normalised top and top pair rapidity distributions, for which no tension was apparent. Additionally, the sensitivity in the high $m_{t \bar{t}}$ region, where beyond-the-standard-model effects may enter, is smaller. A clear impact on the gluon PDF at high $x$, comparable to the effect of inclusive jet data, was demonstrated, as shown in Fig. 5 (left). The CMS collaboration has also recently explored the impact of double-differential $t \bar{t}$ measurements as presented in [12, 39], although here the theoretical calculation is only available at NLO so far. ATLAS presented a study that correlates $t \bar{t}$ and $Z$ production in $p p$ collisions at various $\sqrt{s}=7,8,13 \mathrm{TeV}$ to improve the impact on PDF constraints $[24,40]$.

In $[37,42]$ the impact of measurements of the $Z$-boson transverse momentum distribution on the determination of PDFs was explored. The theoretical calculations are now available at up to 

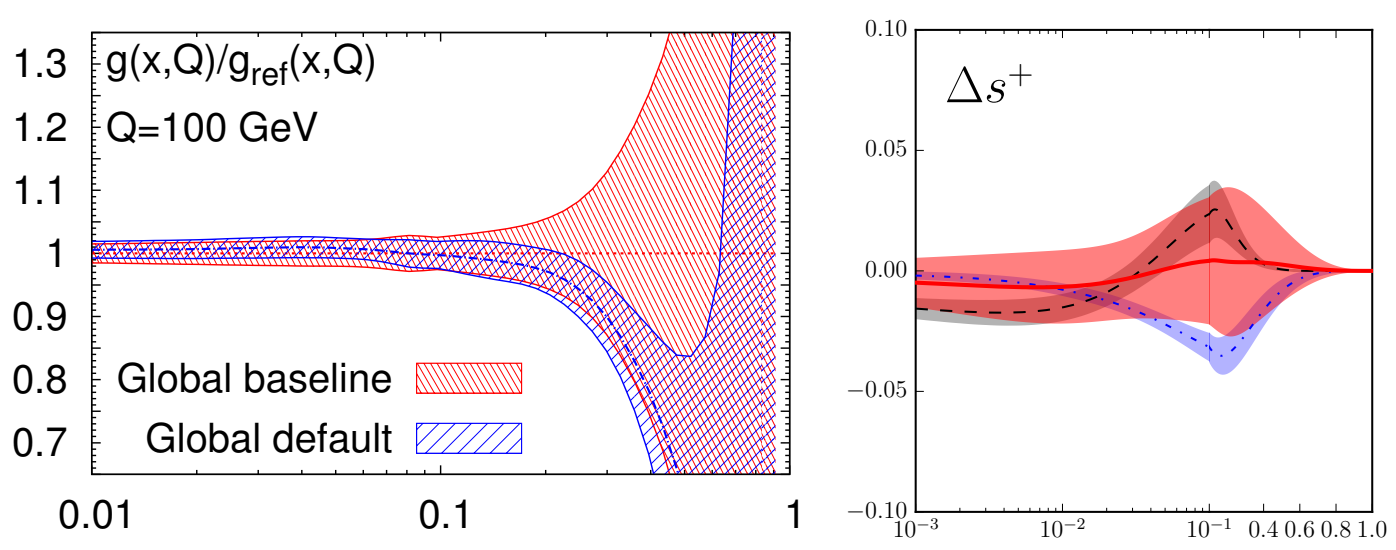

Figure 5: (Left) Impact on the gluon PDF with respect to the global baseline of ATLAS and CMS differential top production data at NNLO [38]. The "optimal" fit corresponds to the recommended choice of observables described in the text. (Right) The polarised strangeness $\Delta s^{+}$distribution extracted from the JAM17 preliminary combined fit to polarised PDFs and fragmentation functions [41].

NNLO in QCD and NLO in EW [43]. To achieve a good description of the data, an additional 1\% of bin-to-bin uncorrelated uncertainty was included, which it was argued was necessary to account for statistical uncertainties on the theory calculations and a possible underestimation of experimental uncertainties. The impact of these ad-hoc uncertainties on the extracted PDFs was found to be relatively minor. The analysis found some tension when including the normalised distributions, e.g. the ATLAS $7 \mathrm{TeV}$ data. This is believed to be a feature of either the treatment of the experimental correlations or the theory, in the case of normalised distributions, where both the high $p_{\mathrm{T}}^{Z}$ region and the integral over the full distributions enter the fit. A fit to the $8 \mathrm{TeV}$ unnormalised distributions was found to give a significant reduction in PDF uncertainties in comparison to a HERA-only PDF set.

Progress in the determination of polarised PDFs was also presented. In $[41,44]$ the first simultaneous extraction of both polarised PDFs and fragmentation functions from DIS, semi-inclusive DIS (for which the pion and kaon fragmentation functions are required), and semi-inclusive annihilation data at NLO within the JAM17 framework was discussed. To achieve this, an iterative Monte Carlo fitting method was used. A good description of the data was achieved, and as shown in Fig. 5 (right), a $\Delta s^{+}$consistent with zero across the entire $x$ range within fairly large uncertainties was found, in contrast to some earlier studies. In $[45,46]$ a theoretical calculation of the transverse momentum dependent PDFs within the light-front quark di-quark model was presented. The resultant PDFs were found to be consistent with global fits, and were able to successfully describe the Collins asymmetry in semi-inclusive DIS.

The determination of the intrinsic charm content of the proton was discussed in [47, 5]. In most PDF analyses the charm PDF is generated extrinsically from perturbative $g \rightarrow c \bar{c}$ splittings, and is therefore determined from the other PDFs with no additional freedom. However, the possibility of some intrinsic non-perturbative charm content in addition to this extrinsic content has been a subject of debate for some time. Two different approaches to this problem were presented here. In a CT study $[47,48]$ physically motivated models for sea- and valence-like components were considered. While these models determined the intrinsic charm $x$ dependence, the normal- 

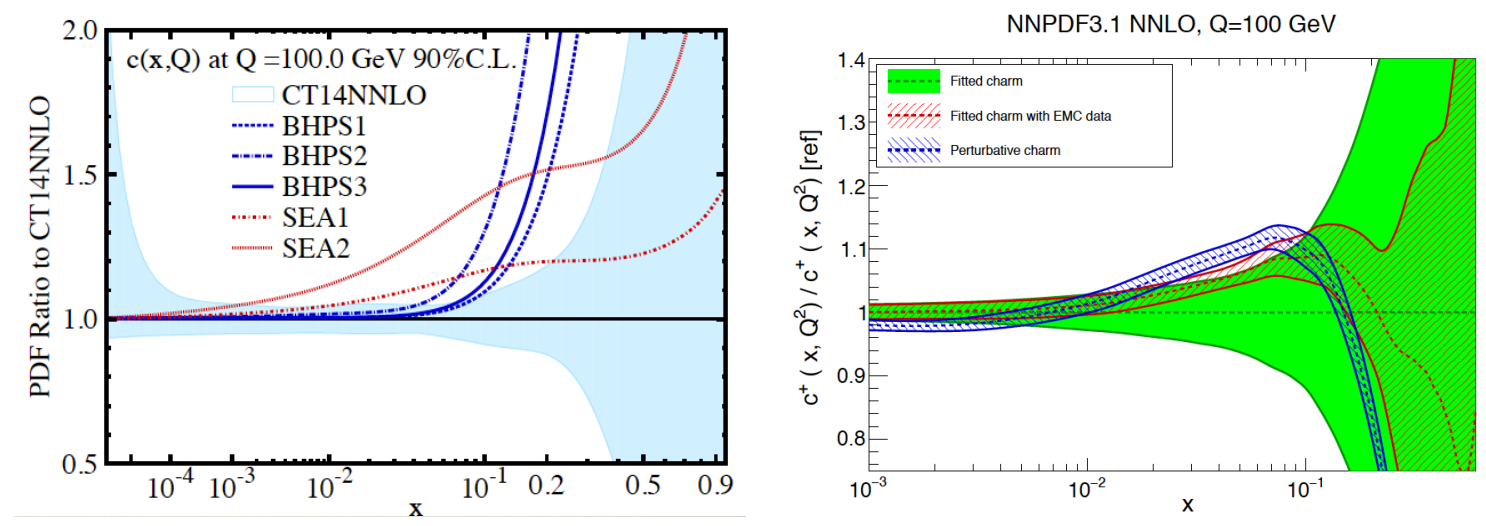

Figure 6: (Left) Ratio of the CT14 charm quark PDF with different model fits of intrinsic charm, to the baseline extrinsic only PDF [47]. (Right) Ratio of the NNPDF fitted charm quark PDF, including and excluding the EMC data in the fit, to the baseline extrinsic only PDF [5]. In both cases the scale $Q=100 \mathrm{GeV}$ is taken.

isation was left free and fitted to data. Alternatively, in the NNPDF study [5, 49] a completely agnostic approach was taken and the charm PDF was fitted in the same fashion as the light quarks. Excluding the early EMC data, for which questions have been raised with respect to the reliability of the experimental systematic uncertainties, both studies place a limit on the momentum fraction carried by intrinsic non-perturbative charm of 1(2)\% for NNPDF (CT). Including the EMC data, NNPDF finds evidence for non-perturbative charm at the $1.5 \sigma$ level. Future $Z+c$, open-charm, and $J / \psi$ measurements from the LHC should help further constrain or discover intrinsic charm. The extracted charm quark PDFs are shown in Fig. 6.

In [50] the resummation of $\log ^{k}(x)$ terms in the QCD splitting functions, which are particularly important at low $x$, was presented. This was applied for the first time within a PDF fit to a range of fixed-target and HERA DIS data. Interestingly, some improvement in the description, in particular at NNLO, was found. The impact on the PDFs at low $x$ is found to be quite large, and a $\sim 1 \%$ change in the Higgs gluon-fusion production cross section is observed. However, this can only be taken as an indication of possible effects, as low $x$ resummation should also be included in the cross-section calculations themselves, which may reduce the effect. The outlook for including further processes in the context of a more global fit was discussed, and has subsequently been presented in [51].

\section{Tools for PDF fits}

In recent years there has been a large increase in theory calculations for standard-candle processes at NNLO in perturbative QCD. These now cover, in addition to inclusive $W / Z / \gamma^{*}$ production, various $2 \rightarrow 2$ processes, e.g. $W / Z+$ jet [52, 53], $t \bar{t}$ [54] and inclusive jets [55] in both $e p$ and $p p$ collisions. However, each calculation requires a large computational effort and thus they are not directly usable in PDF fits. The APPLfast project [56] works on extending the grid-technology for NLO calculations known from FastNLO and APP Lgrid $[57,58]$ to the calculations provided by the NNLOJET program. While significant computations of about 200,000 CPU-hours must be 

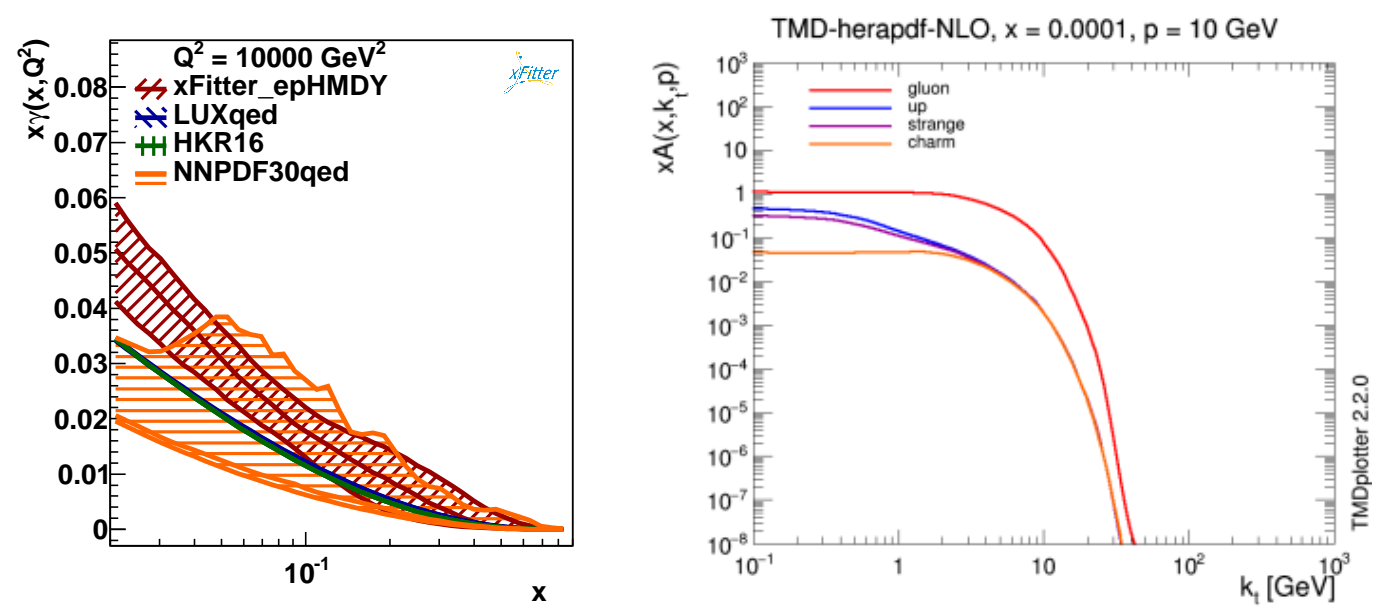

Figure 7: (Left) Extraction of the photon PDF from ATLAS high-mass Drell-Yan data [64, 65]. (Right) Transverse momentum dependent PDFs evolved using the parton branching model [66].

performed initially, any subsequent convolution with an arbitrary PDF is completed in much less than one second. Recently the work on fastNLO tables for NNLO $t \bar{t}$ calculations has been completed [59]. The availability of fast NNLO calculations will be an important ingredient to improve the precision of PDF fits with hadron-collider data.

The APFEL package $[60,61]$ is a library for the computation of collinear PDF evolution and DIS structure functions. In the current version, the package supports evolution at up to NNLO in QCD and NLO in QED, various options for heavy-quark contributions (e.g. fixed/variable-flavournumber schemes, pole masses, $\overline{\mathrm{MS}}$ masses), and a fast computation of DIS observables. APFEL is the main evolution code used by the NNPDF collaboration [6] and is also available in the XFitter package [62, 63]. APFEL features flexible interfaces to Fortran, $\mathrm{C} / \mathrm{C}++$, and Python code.

Finally, the $x F$ itter package $[62,63]$ is the major platform used for PDF fits and analyses outside the dedicated PDF collaborations. It is open source and integrates several other programs that are necessary to perform a PDF analysis. The xFitter team has recently developed a new major release, 2.0.0, with significant improvements. There are more than 30 public results obtained with XFitter since the start of the project. Members of the LHC experiments are currently the main developers and users of $x F i t t e r$, using the program to assess data sensitivity to PDFs and compatibility with QCD predictions. Two recent results by the XFitter team were featured at this workshop. The first was an extraction of the photon PDF using high-mass Drell-Yan data from the ATLAS experiment at the LHC [64, 65]. The second result explored a new approach to performing the evolution of transverse momentum dependent PDFs using the parton branching model [66]. These two results are showcased in Fig. 7 (left) and (right), respectively.

\section{PDFs and effects in heavy Nuclei}

The study of PDFs for heavy atomic nuclei continues to be an interesting topic with a steady level of activity. As has been known for some time, a nucleus is more than the sum of free nucleons, due to nuclear dynamics. Recent new data on heavy ion collisions have increased both the interest in predictions and expanded the available datasets. Traditionally $v N$ scattering has been 

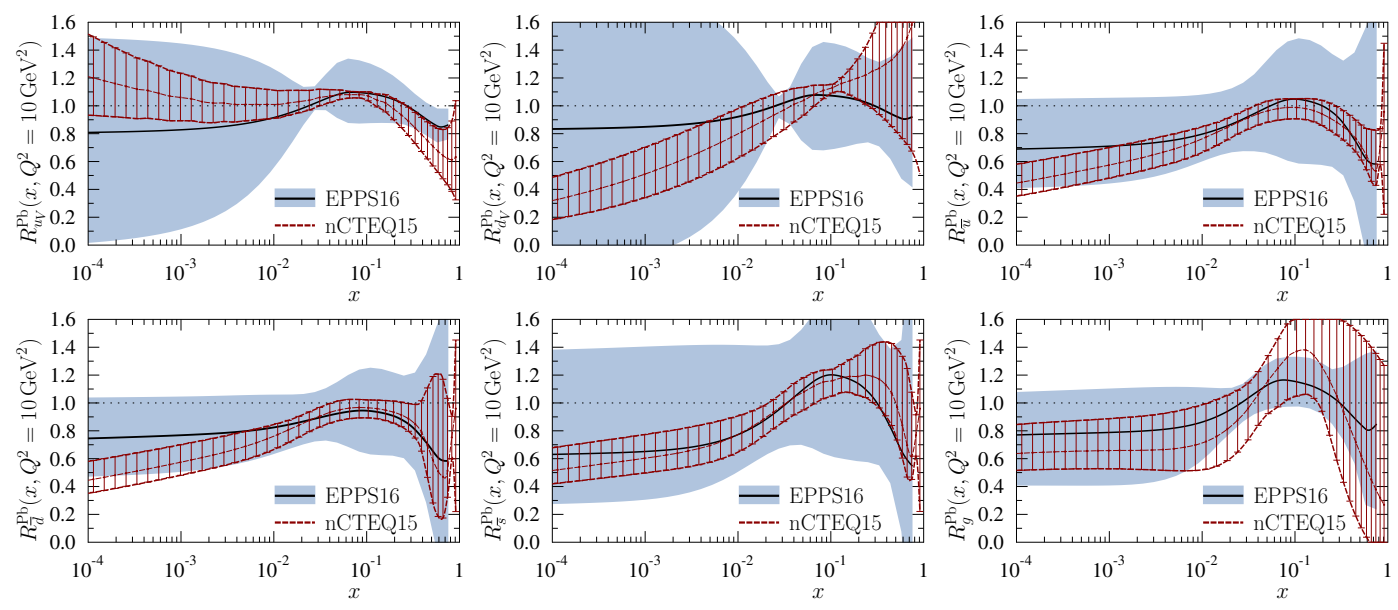

Figure 8: A comparison of the nuclear correction functions for $\mathrm{Pb}$ nuclei as determined in the recent nCTEQ15 and EPPS16 analyses [70].

an important source of information on the strange-quark density, which requires an understanding of the nuclear corrections, as discussed in [67]. While the EMC effect has been known since 1983, work is ongoing to describe this theoretically as a combination of several effects, including dynamic short range correlations, as discussed in [68].

The extraction of nuclear PDFs typically proceeds with a model to parameterise the nuclear effects. The new EPPS16 fit was presented in [69, 70, 71], which relies on a parametrisation of these effects that is fitted to data. For the first time, this fit includes LHC data, which has allowed the parametrisation to be more flexible to reduce the impact of initial assumptions. The inclusion of pion-nucleus Drell-Yan data show some sensitivity to nuclear valence-quark modifications. The approach by the nCTEQ collaboration was presented in [67], which features a generalised $A$ dependent parameterisation of nuclear effects. Fig. 8 shows an overlay of the most recent EPPS and nCTEQ results regarding the modification factor for lead nuclei. A general agreement on the central values can be seen. However, the uncertainties are large and their estimation is typically driven by model choices rather than the data. The Kulagin-Petti approach was presented as well in [72], which uses a microscopic model incorporating Fermi motion.

Another topic of continued interest is the combination $\bar{d}-\bar{u}$ at $x \approx 0.1$, which was found to be significantly larger than zero by the E866 Drell-Yan experiment. Explanations for this effect were discussed in $[73,74]$. These typically use models based on pion exchange, which can be obtained from QCD and calculated in chiral effective field theory. New data by the SeaQuest collaboration has recently disfavoured a possible change of sign change at $x>0.3$, as shown in Fig. 9 (left). In the future, leading neutron analyses and Drell-Yan data with pion beams will constrain pion PDFs at low and high $x$ in an upcoming neutron tagged DIS experiment at JLAB [75].

Several LHC measurements sensitive to nuclear effects were presented by the experiments [76, 77] or discussed further by the fit collaborations $[69,72,67]$. These included most prominently data on the production of jets or $W / Z$ bosons in LHC $p \mathrm{~Pb}$ collisions. Comparisons of jet production between $p p$ and $p \mathrm{~Pb}$ collisions have been made, and while they are similar when integrated in 

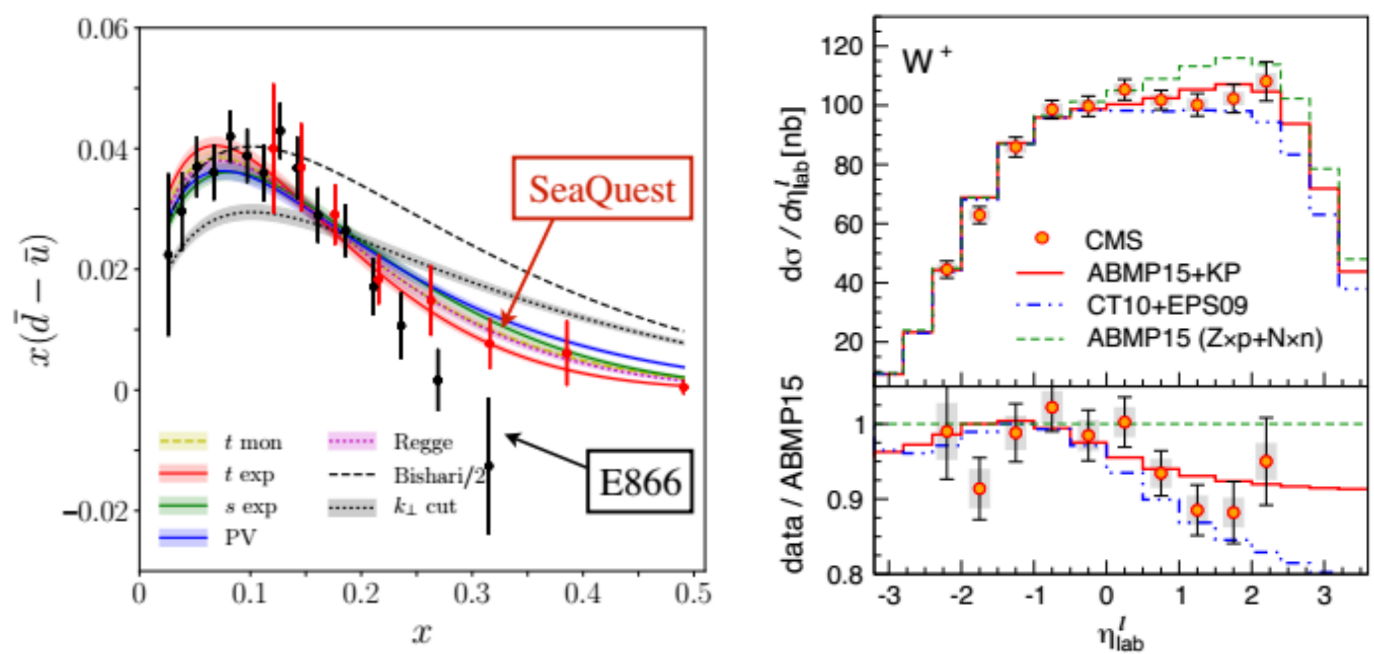

Figure 9: (Left) Data from the E866 and SeaQuest experiments on $\bar{d}-\bar{u}$ [73]. (Right) An example of $p \mathrm{~Pb}$ LHC data, here $W^{+}$production, that is used to differentiate between nuclear models and constrain model parameters [72].

rapidity, CMS data show significant modification as a function of jet rapidity. Indeed, this data already provides some constraints on the EPPS16 fit. An example of data on $W^{+}$production in $p \mathrm{~Pb}$ collisions compared to a variety of predictions is shown in Fig. 9 (right); not all nuclear modifications improve the description of the $W^{ \pm}$and $Z$ data. Similar to the high precision $p p$ case, correlations in these $p \mathrm{~Pb}$ data are starting to be exploited, e.g. by analysing the forward/backward and $W^{+} / W^{-}$asymmetries. More precise measurements in the near future will significantly improve our knowledge. New $v N$ cross-section data is expected in the near future from the MINERvA experiment [78], although the reach in $Q^{2}$ will be limited.

\section{New Theory Developments}

A number of new theoretical ideas and developments were also discussed at the conference. In [81] a summary of the first joint PDF and lattice QCD workshop, which took place in Oxford, March 2017, was presented. The aim is to explore the possibility for lattice QCD calculations to be used as constraints for global PDF fits. Many topics were discussed at this workshop, including the need to identify benchmarks that are well constrained by global fits and can therefore be used to validate the lattice inputs, the choice of observables that could benefit from lattice constraints, and the understanding of various sources of systematic uncertainty in the lattice calculations. This has subsequently been summarised in the form of a white paper [82], which serves as a basis for future work in the area.

In [79, 83], a novel approach to performing PDF fits was described. Markov Chain Monte Carlo techniques were used to extract the probability density functions of PDFs, allowing for further information on the PDF uncertainties. The minimisation was performed using a hybrid (or Hamiltonian) Monte Carlo technique, first applied in lattice QCD applications. The implementation used the xFitter package and a validation was performed using the HERA DIS dataset 

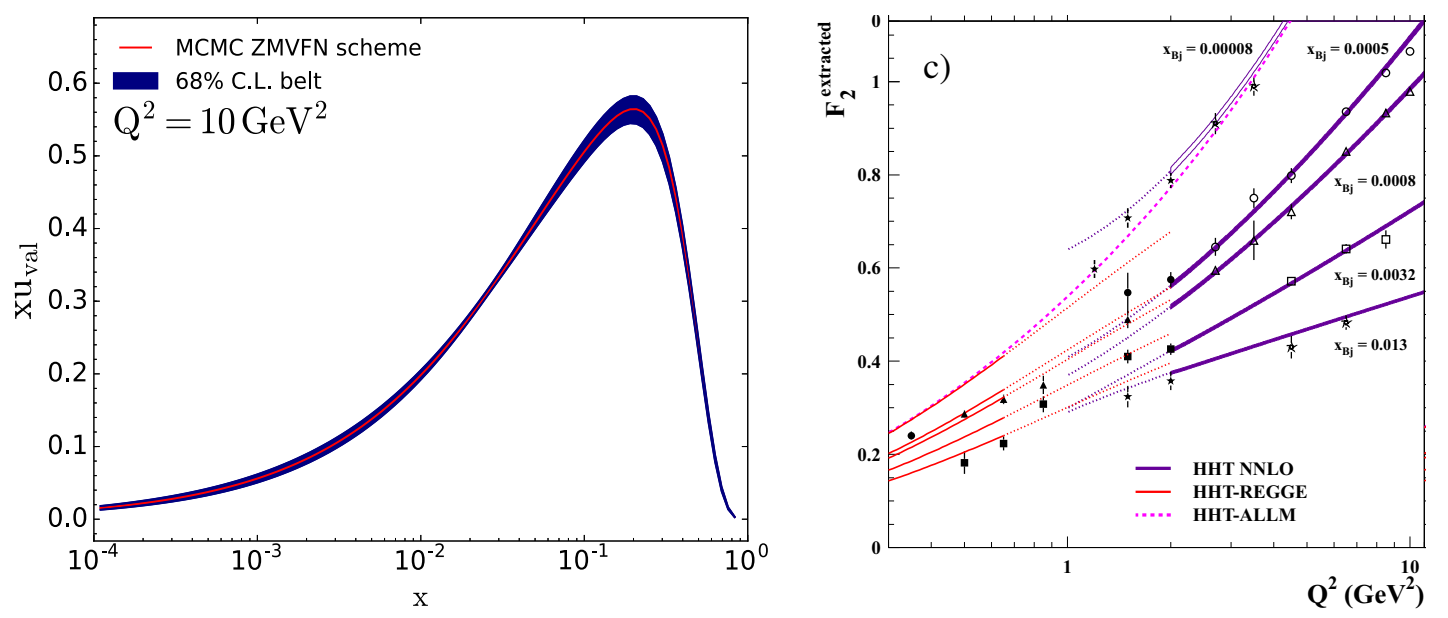

Figure 10: (Left) Up valence quark distribution extracted using the Markov Chain Monte Carlo technique [79]. (Right) The structure function $F_{2}\left(Q^{2}\right)$ for selected values of $x_{B j}$, compared to the Regge and NNLO DGLAP based predictions [80].

with the zero-mass variable-flavour number scheme. The results were found to be consistent with the corresponding HERAPDF set, thus serving as a proof-of-concept. The extracted $u$-valence is shown in Fig. 10 (left).

In $[84,85]$ the determination of CTEQ-TEA MC replica sets was discussed. Alternative techniques for converting from the Hessian to MC PDFs were considered, either sampling directly in the PDF $f$, or in $\ln f$. The latter case guarantees PDF positivity, provided the original Hessian set is positive within its uncertainty. In addition, a shift is introduced to guarantee that the central value of the MC replicas corresponds exactly to the central Hessian set, something which is otherwise not guaranteed. The MC sets using these techniques were compared to the original Hessian PDFs, and found to agree well. These are now publicly available in LHAPDF format.

In $[86,87]$, a new calculation of certain contributions to the 4-loop splitting functions was discussed. In particular, a lattice basis reduction technique was applied to reconstruct the large $n_{F}$ contributions to the splitting functions from the calculated Mellin moments. These results have been validated against a range of known results in various limiting regions, and are an important step towards a consistent treatment of PDFs at $\mathrm{N}^{3} \mathrm{LO}$.

The description of HERA DIS data in the low $x$ and $Q^{2}$ region was discussed in two talks [88, 89]. In [89, 80] the HERA data was fit using a two-component approach, with standard perturbative QCD DGLAP applied above a $Q^{2}$ of $2 \mathrm{GeV}^{2}$, and a Regge-inspired model applied below. The data agrees well with these approaches in their expected regions of validity, but not in the transition region. However, as shown in Fig. 10 (right) the data shows a smooth transition between the two regions, prompting the conclusion that nature does not "know" about perturbation theory. In [88, 90] a QCD-inspired model of higher-twist corrections was used to supplement standard DGLAP evolution, with the parameters of these corrections determined in a simultaneous fit to the HERA data. A large improvement in the fit quality at low $Q_{\min }^{2}$ was found upon the inclusion of these corrections, providing possible evidence for the important contribution of higher twists at HERA. 


\section{Conclusions}

The structure of hadrons, specifically PDF dynamics, is an interesting topic that continues to attract attention from the larger nuclear and particle physics communities. From a practical perspective, experiments at the current and future energy frontiers using hadron beams need PDF information to exploit their data. Without improved DIS data, much of the attention has now turned to data from the LHC experiments, with a continuous effort to measure and predict standard-candle processes at the highest possible precision, and include these data into global PDF fits. Many of the talks in this working group revolved around these topics. For the case of heavy nuclei the range of datasets is more scarce than for protons, giving the new data from LHC and elsewhere a more prominent role.

Beyond the topic of the LHC data and applications, fundamental topics raise interest, including the validity of the framework of collinear factorisation, the treatment of heavy-quark contributions, non-perturbative effects and techniques, resummation effects, and the nature of PDFs at very small or large $x$.

Many of these topics were debated lively in the working groups sessions, demonstrating that the DIS conference is one of the focal points of the community for these discussions.

\section{Acknowledgements}

We would like to thank all the speakers and participants that contributed to the success of the working group 1 programme with interesting presentations and lively discussions. We also thank the organisers for a well-organised and interesting DIS 2017 conference, and for giving us the opportunity to convene this session.

\section{References}

[1] A. Giammanco, R. Gauld, and A. Pearce, Working Group 5: Physics with Heavy Flavours, in 25th International Workshop on Deep-Inelastic Scattering and Related Topics (DIS 2017) Birmingham, UK, April 3-7, 2017, 2017, 1710.04069.

[2] Alberto Belloni, Tim Martin, Marco Zaro, WG4 summary presentation at this conference.

[3] Jun Gao, in presentation at this conference.

[4] R. S. Thorne, L. A. Harland-Lang, and A. D. Martin, Inclusion of new LHC data in MMHT PDFs, in 25th International Workshop on Deep Inelastic Scattering and Related Topics (DIS 2017) Birmingham, UK, April 3-7, 2017, 2017, 1708.00047.

[5] Juan Rojo, in presentation at this conference.

[6] NNPDF, R. D. Ball et al., Eur. Phys. J. C77, 663 (2017), 1706.00428.

[7] Philip Sommer, in presentation at this conference.

[8] ATLAS, M. Aaboud et al., Eur. Phys. J. C77, 367 (2017), 1612.03016.

[9] H1, V. Andreev et al., Eur. Phys. J. C77, 215 (2017), 1611.03421.

[10] Daniel Britzger, in presentation at this conference. 
[11] Jeff Dandoy, in presentation at this conference.

[12] Katerina Lipka, in presentation at this conference.

[13] Engen Eren, in presentation at this conference.

[14] ATLAS, M. Aaboud et al., JHEP 09, 020 (2017), 1706.03192.

[15] CMS, V. Khachatryan et al., JHEP 03, 156 (2017), 1609.05331.

[16] ATLAS, G. Aad et al., JHEP 02, 153 (2015), 1410.8857, [Erratum: JHEP09,141(2015)].

[17] L. A. Harland-Lang, A. D. Martin, and R. S. Thorne, (2017), 1711.05757.

[18] CMS, A. M. Sirunyan et al., Eur. Phys. J. C77, 746 (2017), 1705.02628.

[19] CMS Collaboration, Determination of the strong coupling constant from the measurement of inclusive multijet event cross sections in pp collisions at $\sqrt{s}=8 \mathrm{TeV}, 2017$, CMS-PAS-SMP-16-008, https://cds.cern.ch/record/2253091.

[20] Benoit Roland, in presentation at this conference.

[21] CMS, A. M. Sirunyan et al., (2017), 1711.02143.

[22] LHCb, R. Aaij et al., Phys. Lett. B767, 110 (2017), 1610.08142.

[23] LHCb, R. Aaij et al., JHEP 10, 030 (2016), 1608.01484.

[24] Markus Zinser, in presentation at this conference.

[25] ATLAS, G. Aad et al., JHEP 08, 009 (2016), 1606.01736.

[26] Marcin Kucharczyk, in presentation at this conference.

[27] LHCb, R. Aaij et al., JHEP 09, 136 (2016), 1607.06495.

[28] Salvatore Fazio, in presentation at this conference.

[29] Claudia Glasman, in presentation at this conference.

[30] ATLAS, M. Aaboud et al., Phys. Lett. B770, 473 (2017), 1701.06882.

[31] J. M. Campbell, R. K. Ellis, and C. Williams, Phys. Rev. Lett. 118, 222001 (2017), 1612.04333.

[32] Juan Rojo, in presentation at this conference.

[33] Stephen Jones, in presentation at this conference.

[34] R. Gauld and J. Rojo, Phys. Rev. Lett. 118, 072001 (2017), 1610.09373.

[35] S. P. Jones, A. D. Martin, M. G. Ryskin, and T. Teubner, J. Phys. G44, 03LT01 (2017), 1611.03711.

[36] Emanuele Nocera, in presentation at this conference.

[37] Maria Ubiali, in presentation at this conference.

[38] M. Czakon, N. P. Hartland, A. Mitov, E. R. Nocera, and J. Rojo, JHEP 04, 044 (2017), 1611.08609.

[39] CMS, A. M. Sirunyan et al., Eur. Phys. J. C77, 459 (2017), 1703.01630.

[40] ATLAS, M. Aaboud et al., JHEP 02, 117 (2017), 1612.03636.

[41] Jacob Ethier, in presentation at this conference.

[42] E. R. Nocera and M. Ubiali, Constraining the gluon PDF at large x with LHC data, 2017, 1709.09690. 
[43] R. Boughezal, A. Guffanti, F. Petriello, and M. Ubiali, JHEP 07, 130 (2017), 1705.00343.

[44] J. J. Ethier, N. Sato, and W. Melnitchouk, Phys. Rev. Lett. 119, 132001 (2017), 1705.05889.

[45] Tanmay Maji, in presentation at this conference.

[46] T. Maji and D. Chakrabarti, Phys. Rev. D95, 074009 (2017), 1702.04557.

[47] Marco Guzzi, in presentation at this conference.

[48] T.-J. Hou et al., (2017), 1707.00657.

[49] NNPDF, R. D. Ball et al., Eur. Phys. J. C76, 647 (2016), 1605.06515.

[50] Luca Rottoli on behalf of Marco Bonvini, in presentation at this conference.

[51] R. D. Ball et al., (2017), 1710.05935.

[52] R. Boughezal et al., Phys. Rev. Lett. 116, 152001 (2016), 1512.01291.

[53] A. Gehrmann-De Ridder, T. Gehrmann, E. W. N. Glover, A. Huss, and T. A. Morgan, Phys. Rev. Lett. 117, 022001 (2016), 1507.02850.

[54] M. Czakon, D. Heymes, and A. Mitov, Phys. Rev. Lett. 116, 082003 (2016), 1511.00549.

[55] J. Currie, E. W. N. Glover, and J. Pires, Phys. Rev. Lett. 118, 072002 (2017), 1611.01460.

[56] Claire Gwenlan, in presentation at this conference.

[57] T. Kluge, K. Rabbertz, and M. Wobisch, FastNLO: Fast pQCD calculations for PDF fits, in Deep inelastic scattering. Proceedings, 14th International Workshop, DIS 2006, Tsukuba, Japan, April 20-24, 2006, pp. 483-486, 2006, hep-ph/0609285.

[58] T. Carli et al., Eur. Phys. J. C66, 503 (2010), 0911.2985.

[59] M. Czakon, D. Heymes, and A. Mitov, (2017), 1704.08551.

[60] V. Bertone, APFEL++: A new PDF evolution library in $\mathrm{C}++$, in 25th International Workshop on Deep Inelastic Scattering and Related Topics (DIS 2017) Birmingham, UK, April 3-7, 2017, 2017, 1708.00911.

[61] V. Bertone, S. Carrazza, and J. Rojo, Comput. Phys. Commun. 185, 1647 (2014), 1310.1394.

[62] S. Alekhin et al., Eur. Phys. J. C75, 304 (2015), 1410.4412.

[63] xFitter Developers' Team, V. Bertone et al., PoS DIS2017, 203 (2017), 1709.01151.

[64] F. Giuli, The photon PDF from high-mass Drell Yan data at the LHC using xFitter, in 25th International Workshop on Deep Inelastic Scattering and Related Topics (DIS 2017) Birmingham, UK, April 3-7, 2017, 2017, 1705.08201.

[65] xFitter Developers' Team, F. Giuli et al., Eur. Phys. J. C77, 400 (2017), 1701.08553.

[66] Ola Lelek, in presentation at this conference.

[67] Fred Olness, in presentation at this conference.

[68] Or Hen, in presentation at this conference.

[69] K. J. Eskola, P. Paakkinen, H. Paukkunen, and C. A. Salgado, The EPPS16 nuclear PDFs, in 25th International Workshop on Deep Inelastic Scattering and Related Topics (DIS 2017) Birmingham, UK, April 3-7, 2017, 2017, 1709.08347. 
[70] K. J. Eskola, P. Paakkinen, H. Paukkunen, and C. A. Salgado, Eur. Phys. J. C77, 163 (2017), 1612.05741 .

[71] F. Arleo, E. Chapon, and H. Paukkunen, Features of $\mathrm{W}$ production in p-p, p- $\mathrm{Pb}$ and $\mathrm{Pb}-\mathrm{Pb}$ collisions, in 25th International Workshop on Deep Inelastic Scattering and Related Topics (DIS 2017) Birmingham, UK, April 3-7, 2017, 2017, 1709.08351.

[72] Roberto Petti, in presentation at this conference.

[73] Wallny Melnitchouk, in presentation at this conference.

[74] P. Paakkinen, K. J. Eskola, and H. Paukkunen, Pion-nucleus Drell-Yan data as a novel constraint for nuclear PDFs, in 25th International Workshop on Deep-Inelastic Scattering and Related Topics (DIS 2017) Birmingham, UK, April 3-7, 2017, 2017, 1710.05736.

[75] Cynthia Keppel, in presentation at this conference.

[76] Andre Mischke, in presentation at this conference.

[77] Emilien Chapon, in presentation at this conference.

[78] Marianette Wospakrik, presentation at this conference.

[79] Mariane Mangin-Brinet, in presentation at this conference.

[80] I. Abt et al., Phys. Rev. D96, 014001 (2017), 1704.03187, [Phys. Rev.D96,014001(2017)].

[81] E. R. Nocera, H.-W. Lin, F. Olness, K. Orginos, and J. Rojo, The PDFLattice2017 workshop: a summary report, in 25th International Workshop on Deep Inelastic Scattering and Related Topics (DIS 2017) Birmingham, UK, April 3-7, 2017, 2017, 1709.01511.

[82] H.-W. Lin et al., (2017), 1711.07916.

[83] Y. G. Gbedo and M. Mangin-Brinet, Phys. Rev. D96, 014015 (2017), 1701.07678.

[84] Jun Gao, in presentation at this conference.

[85] T.-J. Hou et al., JHEP 03, 099 (2017), 1607.06066.

[86] J. Davies, The Large- $n_{f}$ Limit of the Four-Loop Splitting Functions in QCD, in 25th International Workshop on Deep Inelastic Scattering and Related Topics (DIS 2017) Birmingham, UK, April 3-7, 2017, 2017, 1709.00368.

[87] J. Davies, A. Vogt, B. Ruijl, T. Ueda, and J. A. M. Vermaseren, Nucl. Phys. B915, 335 (2017), 1610.07477.

[88] Leszek Motyka, in presentation at this conference.

[89] Katarzyna Wichmann, in presentation at this conference.

[90] L. Motyka, M. Sadzikowski, W. Slominski, and K. Wichmann, (2017), 1707.05992. 